\title{
A NOVEL METHOD OF CLOUD AND SHADOW EXTRACTION WITH MULTI-TEMPORAL LANDSAT8 OLI IMAGES
}

\author{
Yuan.Ni ${ }^{\text {a,b }}$,Guojin.He ${ }^{\text {a,d,e* }}$, Wei.Jiang ${ }^{\text {a,c }}$ \\ ${ }^{a}$ Institute of Remote Sensing and Digital Earth, Chinese Academy of Sciences, Beijing, China, jiangwei@radi.ac.cn \\ ${ }^{b}$ Key Laboratory of Spatial Data Mining \& Information Sharing of Ministry of Education, Spatial Information Research Center of Fu \\ jian Province, Fuzhou University, Fuzhou, China, niyuanirsa@hotmail.com \\ ${ }^{\mathrm{c}}$ University of the Chinese Academy of Sciences, Beijing, China \\ ${ }^{\mathrm{d}}$ Key Laboratory of Earth Observation Hainan Province, Sanya, China, hegj@radi.ac.cn \\ ${ }^{\text {e }}$ Sanya Institute of Remote Sensing, Sanya, China
}

KEY WORDS: Cloud, Shadow, Brightness difference, Multi-temporal, LandsatlOLI.

\begin{abstract}
Cloud and Shadow removal is a significant step in remote sensing image process. As we all know, the ground object coverage type of the same area of the remote sensing image has little change in the short term. But for cloud and shadow coverage areas, the ground object coverage type has large change. Therefore, according to the difference between the two Landsat / OLI images caused by changes in the cover, this paper presents a method of extracting clouds and shadows based on differences in luminance values. This method selects two thresholds for the difference of brightness values, and extracts the clouds and shadows respectively, and validates them with random point method, which can obtain high precision of extracting cloud and shadow and satisfy the actual application needs.
\end{abstract}

* Corresponding author 


\section{INTRODUCTION}

In remote sensing images, the similarity between cloud and shadow spectral information and other features, and the uncertainty of objects distribution caused by the presence of clouds and shadows, have brought difficulties to identify and classify objects. Thus, the removal of cloud and shadow to reproduce the clear features in remote sensing images, which is of great significance for the extraction of objects(Sunyurp.P2013,Luo Y,2008).

Removing the clouds and shadows of single- temporal remote sensing images mostly by constructing algorithms(Hughes M,2014); Liang D(2012)used support vector machine algorithm for cloud detection, and combined with the sun angle information for cloud shadow detection, Finally, the simulation experiments show the superiority of the method in the effects of removing the cloud and shadow .Zhu Z(2012) presented a cloud detection method based on Fmask (Function of mask) by the object oriented method, first this method distinguishes cloud and non-cloud through the physical characteristics, then cloud shadow position determined by cloud location and angle of the sensor, the precision of this method is high, but the difference effect of cloud and ice is not ideal. Jiang $\mathrm{MM}(2015)$ used the combined band after principal component analysis and transformation to improve the Fmask cloud detection algorithm, which solves the problem that Fmask algorithm is difficult to distinguish between cloud and ice. Removing the clouds and shadows of multi - temporal remote sensing images mostly by reflectance difference; Zhu $\mathrm{Z}$ (2014)made use of multi-temporal images to further reduce the confusion of cloud, cloud shadows and snow on the basis of the Fmask algorithm. Jin S(2013) used the spectral information of two images to extract the cloud and shadow suspicion area, and then used the temporal and spatial relations between cloud and cloud shadow to extract cloud shadow information effectively. This method can use reasonable value instead of cloud and shadow coverage area, but it will appear part of the mixed phenomenon between shadows and terrain shadows, water. Lu $\mathrm{X}$ (2016) through the threshold segmentation to get all the shadows in the image, and then used near time cloud-free image for band operations and morphological filtering to obtain accurate cloud shadow. Mi XT(2016) extracted the cloud shadows by the reflectivity difference between the cloud shadow and the typical objects on similar images. The method requires there is no cloud overlap between the reference image and the target image, and the land cover type has little change.

The spectral characteristics of the cloud are similar to those of snow and ice(Ehrlich A,2017), The spectral characteristics of the shadow are similar to those of the water body(Li S,2013), and the objects covered by the clouds and the shadow are unknown, which are easy to cause misunderstanding and leakage. In order to solve these problems, firstly, the two images of the same region and near time are pre-treatment. According to principle: The cloud and the shadow change into other objects, the brightness value changes greatly, and other objects in a short period of time will not change into other types of objects, the brightness value changes little; the clouds and shadows will be effectively extracted through the use of the brightness difference.

\section{RESEARCH METHOD}

Cloud and shadow are the main factors that interfere with the accuracy of ground objects extraction(Huang C,2010), not only because the spectral curves of other feature is similar to the cloud and shadow, it is difficult to exclude the clouds and shadows through the threshold of a single band, but also in the cloud and shadow coverage area, the optical sensor is difficult to penetrate the clouds to identify the objects(Goodwin NR,2013). Therefore, it is necessary to first exclude the clouds and shadows, and then use the index to extract the features to improve the accuracy of extraction of features(Simpson J,2000).

Firstly, in the official website of United States Geological Survey to download image of the same area and adjacent time, In order to eliminate the effects of clouds and shadows in one of the images, another image needs to meet the conditions of cloudless. The interaction between the reflected radiation energy of the earth surface and the atmosphere of the earth causes the radiation energy to be absorbed or scattered, and the spectral distribution of the radiation is changed, which can not accurately extract the objects based on gray value features. Preprocessing of remote sensing image using 6S atmospheric correction model, and considering the spatial geometric relationship between the sun, the ground target, the sensor and the elevation information of the ground, the radiation error caused by the atmospheric factors in the remote sensing image is eliminated. Secondly, the brightness values of two images are calculated; due to objects of two image of near time have little change, so the difference between the brightness values of the two images is small; the brightness value decreases from the cloud region to the cloudless area; the brightness value increases from the cloud shadow area to the shadowless area;

According to the difference of brightness value, the cloud and cloud shadows were extracted by threshold. Finally, accuracy verification of clouds and shadows is done by selecting random points. The overall flow chart shown in Figure 1.

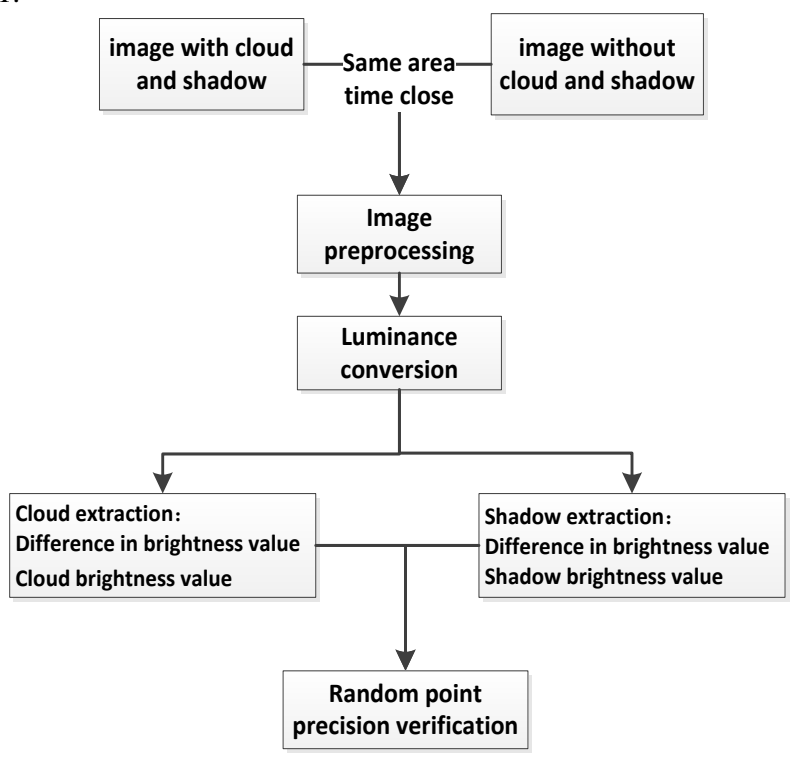

Figure 1.Study flow chart

EXPERIMENT PROCESSING AND ANALYSIS

\section{Data processing}

This paper uses two Landsat8 OLI image data, the data includes data without cloud and shadow on 20 April 2013 and data with cloud and shadow on May 22, 2013. Its data covers two major lakes in Yunnan Province: Dianchi Lake and Fuxian Lake.

First of all, the Landsat8 OLI image data is preprocessed , which is done by $6 \mathrm{~S}$ atmospheric correction model. The model is calculated for each pixel of the lookup table based on the sensitivity analysis of solar zenith angle, water vapor, ozone concentration and aerosol optical thickness. The model is calculated for each pixel of the lookup table based on the 
sensitivity analysis of solar zenith angle, water vapor, ozone concentration and aerosol optical thickness, and get the correction coefficient to modify the surface reflectance image , so as to eliminate the atmospheric effects(Peng Y,2016). As shown in Figure 2.

Various bands of the remote sensing image record the weak difference of the spectrum of the ground objects. If we make full use of the differences of the objects in each band, we can identify the features more effectively(Zhao YS,2013). In this paper, according to the analysis of the main components and correlation, the near infrared, red and green bands (band 543) are transformed into Hue 、 Lightness and Saturation. The change of land cover type will cause the change of the brightness value of different time and phase, through the brightness value of the difference, the cloud and shadow with large changes in the brightness value are extracted separately, and combined with the high luminance values of the cloud area and the low luminance values of the shaded areas to reduce the impact of changes in land cover species. As shown in equation (1) (2).

$$
\begin{aligned}
& L_{\text {nocl oud- }} L_{c l} \text { oud }<\mathrm{T} 1 \\
& L_{c l} \text { oud }>\mathrm{T} 2 \\
& \text { Lnocl oud- }^{-L_{c l} \text { oud }}>\mathrm{T} 3 \\
& \text { Lnocl oud }>\mathrm{T} 4
\end{aligned}
$$

Among them, Lcl oud represents the brightness values of images with cloud and shadow. Lnocl oud represents the brightness values of images without cloud and shadow. $T_{i} \quad(i=1,2,3,4)$ represents the best threshold of the object extraction separately.(1) and (2) extracted the cloud area and shaded area respectively.

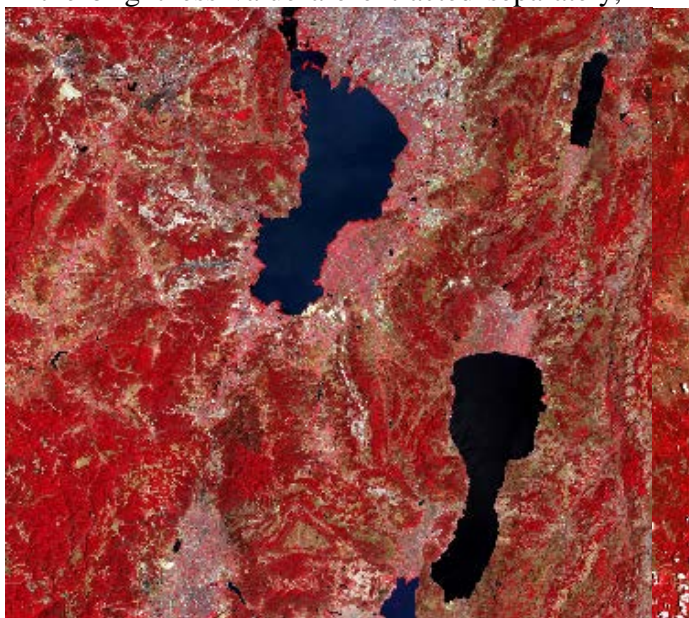

(a)

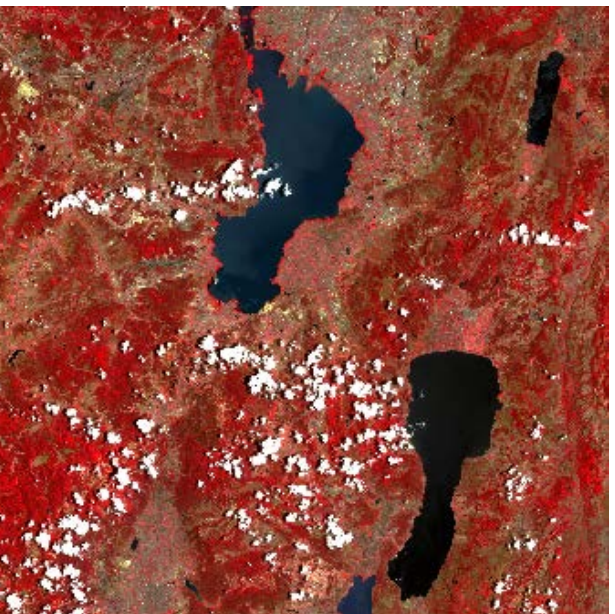

(b)

Figure2 2013.04.20 (a)/2013.05.22 (b) Landsat8 OLI image (Band 543 synthetic display)

In order to determine the threshold of cloud and shadow, a large number of sample points are selected by the method of manual visual interpretation for the brightness value difference image and the cloud and shadow region image. Eventually, we can obtain the maximum brightness difference value of cloud (T1)、 the minimum brightness value of cloud(T2) 、 minimum brightness difference value of shadow(T3)、 the maximum brightness value of shadow(T4), thresholds are shown in Table 1. Two value images of cloud and shadow are covered in 2013.05.22 image. As figure 3. Yellow means that the results of cloud extraction, blue means that the results of shadow extraction.

\begin{tabular}{ccccc}
\hline Threshold & T1 & T2 & T3 & T4 \\
\hline $\begin{array}{c}\text { Optimal } \\
\text { threshold }\end{array}$ & 0 & 0.516 & 0.190 & 0.156 \\
\hline
\end{tabular}

Table1.Threshold extraction results

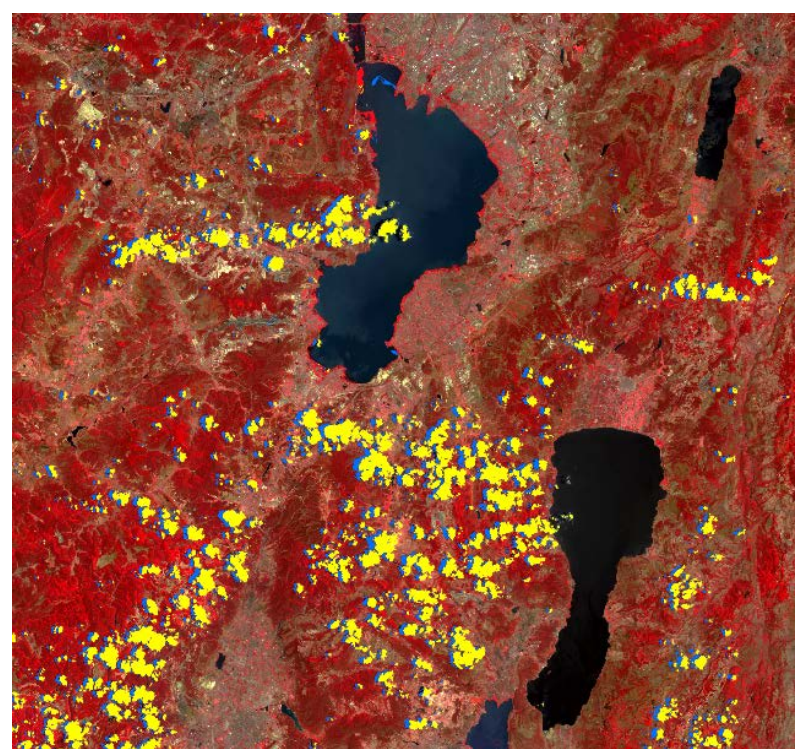

Figure3.Cloud and shadow extraction results

\section{Accuracy verification}

The extracted clouds and shadows are verified using 600 random points. Firstly, remote sensing image with the cloud and shadow is covered by the extracted cloud and shadow, and the generated random points are superimposed on the remote sensing image. Then, the visual interpretation is carried out 
through checking point by point. Error matrix is shown in Table 2 , the accuracy of the extraction of the cloud and shadow is verified by basic precision indicators(Wang $H, 2012$ ), the user accuracy of the cloud is $73 \%$, the mapping accuracy is $100 \%$, and the user accuracy of the shadow is $78 \%$, the mapping accuracy is $86 \%$. The overall accuracy is $96.83 \%$, and the Kappa coefficient is about 0.8449 .

\begin{tabular}{ccccc}
\hline & Cloud & Shadow & Others & Sum \\
\hline Cloud & 38 & 0 & 0 & 38 \\
Shadow & 3 & 18 & 0 & 21 \\
Others & 11 & 5 & 525 & 541 \\
Sum & 52 & 23 & 525 & 600 \\
\hline
\end{tabular}

Table2.Error matrix

\section{Discussion}

In this paper, through the brightness value and the brightness difference value of the two images, we can extract the cloud area and shadow area according to the direct threshold method.

Although the method of extracting cloud and shadow is simple and easy, there are many uncertainties and shortcomings:

(1) The experiment extracts cloud and shadow mainly based on the brightness change of the two images, which requires that the time difference between the two images as small as possible, and the object is almost unchanged. However, in the actual process of image selection, the available image is limited by close time, the minimum interval of Landsat series of images have16 days, if in these days, the earth's surface changes, such as oil spills in seas, algae flooding, new houses, etc., are likely to lead to the mistake of the cloud and the shadow.In the experiment, the time factor in Dianchi Lake lead to changes in the characteristics of the ground objects, the brightness difference value will also change, and therefore the ground objects are used as shadow. As shown in Figure 4 (band 543 synthetic display ). In the future, multi-source data can be used to shorten the time gap and reduce the change of ground objects.

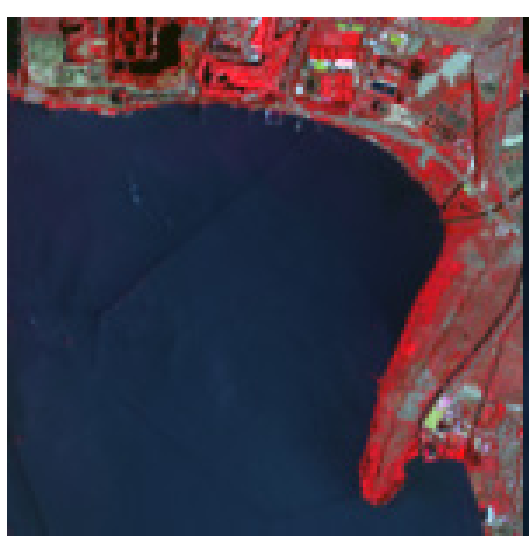

(a)2013.05.22

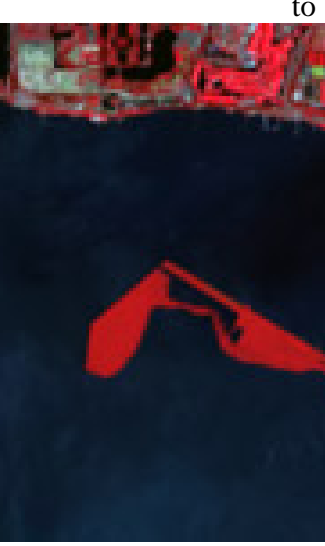

(b)2013.04.20

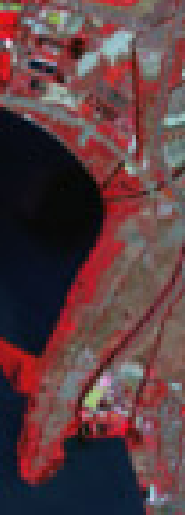

Figure4.False shadow extraction

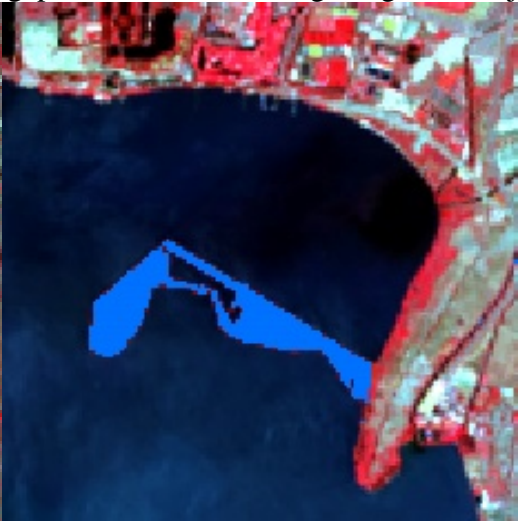

(c) Shadow overlay

(2) Data quality requires one of images as far as possible without interference of cloud and shadow. In the two images, the brightness value has great changes from the cloud or the shadow to other objects, cloud and shadow can be extracted by a specific threshold. But if cloud or shadow in the image is remain cloud or shadow on another image, the change of brightness value is not big, which may lead to the leakage and wrong of ground objects.

(3) The gap between cloud and cloud shadow has both cloud and shadow features, and some also have the characteristics of surface features, it is difficult to be classified as a category, which is often the main cause of error. Although the ground objects in the crevices can have features of the ground objects, it is difficult to extract the ground objects by the features of the experiments, if only the extraction of clouds and shadows, we can through the morphological expansion and corrosion algorithm and will extract the gap which is regarded as a cloud or shadow.

(4) If the cloud shadow in the water body, the change of the brightness value is not big, which makes shadow cannot be extracted completely. As shown in Figure 5(band 543 synthetic display ). In the process of extraction of the shadow may reduce its accuracy, but for an angle, in the process of extraction of water, the cloud and shadow which changes into the water changes little in terms of brightness ,the shadow on the water will no longer be a factor in blocking the water, but can be extracted directly as a water body. ground objects under these circumstances. In future

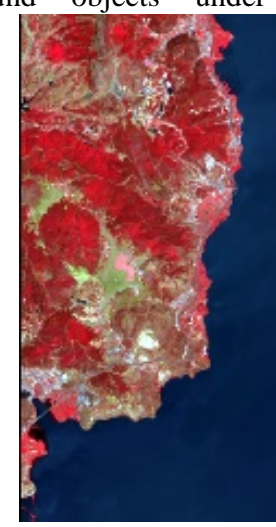

(a)2013.05.22

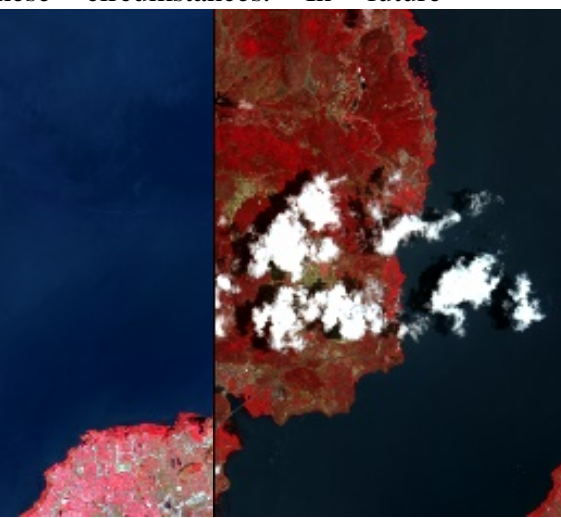

(b) 2013.04.20

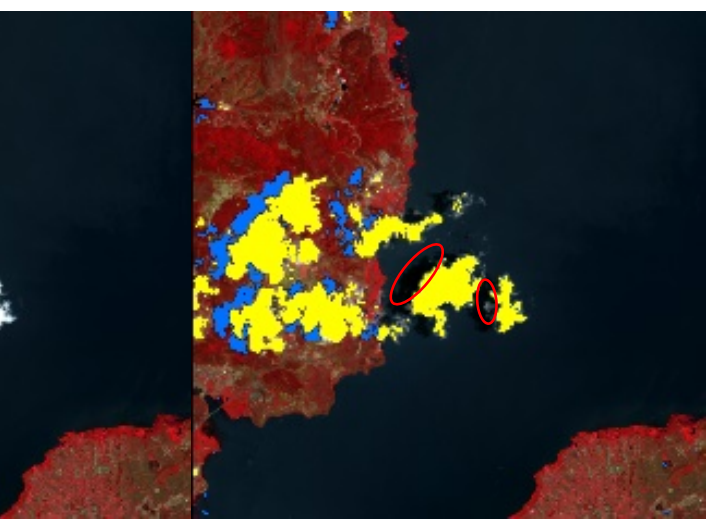

(c) Cloud and Shadow overlay

Figure5. Cloud and Shadow leakage 
According to the principle of change of brightness value, if other remote sensing images, which have the small time interval and almost no change in the ground objects, we can speculate that the maximum brightness changes between the two images must be the place where the cloud and shadow changes to other objects, so the principle should be able to use other remote sensing images, but it is also possible for the small changes in high-resolution images that may cause the huge changes in the brightness value of the entire image(Champion N,2016). So for other images can use the principle of difference between the brightness value of the cloud and shadow extraction, should be discussed in different situations.

\section{CONCLUSION}

Cloud and shadow are the important interference factors of ground objects recognition and classification. In this paper, we take two images of the same region in the same time as an example, Firstly, atmospheric correction is used to eliminate the influence of atmosphere on surface reflectance; Secondly, according to the relationship between the two images of ground objects brightness values, cloud and shadow are extracted; Finally, the random point is used to verify the accuracy. The results show that the above method can effectively carry out cloud and shadow extraction.

In this paper, the cloud and shadow are extracted with high accuracy, but the gap between cloud and shadow is difficult to be classified as a specific category, which requires algorithms such as morphological processing; In the course of the experiment, the artificial selection threshold takes a lot of time and the subjectivity is strong, Future work needs to be studied in automated processing.

\section{ACKNOWLEDGEMENTS}

This research was financially supported by The National Key Research and Development Program of China-rapid production method of large scale global change products (2016YFA0600302), the Hainan Provincial Department of Science and Technology under Grant Nos. ZDKJ2016021 and ZDKJ2016015.

\section{REFERENCES}

Champion N. 2016.Automatic Detection of Clouds and Shadows Using High Resolution Satellite Image Time Series. ISPRS - International Archives of the Photogrammetry, Remote Sensing and Spatial Information Sciences, XLI-B3:475-47

Ehrlich A, Bierwirth E, Istomina L, et al. 2017.Combined retrieval of Arctic liquid water cloud and surface snow properties using airborne spectral solar remote sensing. Atmospheric Measurement Techniques Discussions. 1-25.

Goodwin N R, Collett L J, Denham R J, et al. 2013. Cloud and cloud shadow screening across Queensland, Australia: An automated method for Landsat TM/ETM + time series. Remote Sensing of Environment, 134:50-65.

Huang C, Thomas N, Goward S N, et al. 2010.Automated masking of cloud and cloud shadow for forest change analysis using Landsat images. International Journal of Remote Sensing, 31(20):5449-5464.

Hughes M, Hayes D. 2014. Automated Detection of Cloud and Cloud Shadow in Single-Date Landsat Imagery Using Neural
Networks and Spatial Post-Processing. Remote Sensing, 6(6):4907-4926.

Jiang MM, Hao Z F.2015.Advanced algorithm of PCA-based Fmask cloud detection.Science of Surveying and Mapping,40(2):150-154.

Jin S, Homer C, Yang L, et al. 2013.Automated cloud and shadow detection and filling using two-date Landsat imagery in the USA. International Journal of Remote Sensing, 34(5):1540-1560.

Liang D,Kong J,Hu G S, et al.,2012.The Removal of Thick Cloud and Cloud Shadow of Remote Sensing Image Based on Support Vector Machine.Acta Geodaetica et Cartographica Sinica,41(2):225-231.

Li S, Sun D, Yu Y. 2013.Automatic cloud-shadow removal from flood/standing water maps using MSG/SEVIRI imagery. International Journal of Remote Sensing, 34(15):5487-5502.

Luo Y, Trishchenko A P, Khlopenkov K V. 2008.Developing clear-sky, cloud and cloud shadow mask for producing clear-sky composites at 250-meter spatial resolution for the seven MODIS land bands over Canada and North America. Remote Sensing of Environment, 112(12):4167-4185.

Lu X, Zhao H L, Yang S W ,et al.2016.Detection of thick clouds shadow from Landsat TM data. Science of Surveying and Mapping,41(5).

Mi X T, Sun L, Wei J, et al.2016.Cloud Shadow Detection Algorithm Based on Multi-temporal Remote Sensing Data.Journal of Shandong University of Science and Technology, 35(2):64-72.

Peng Y, He G, Zhang Z, et al. 2016, Study on atmospheric corre ction approach of Landsat8 imageries based on $6 \mathrm{~S}$ model and lo okup table. Journal of Applied Remote Sensing, 10(4):045006.

Simpson J J, Jin Z, Stitt J R. 2000.Cloud shadow detection under arbitrary viewing and illumination conditions. IEEE Transactions on Geoscience \& Remote Sensing, 38(2):972-976.

Sunyurp Park. 2013.Cloud and cloud shadow effects on the MODIS vegetation index composites of the Korean Peninsula. International Journal of Remote Sensing, 34(4):1234-1247.

Wang H, Wang X, Dou A. 2012.Study on the precision evaluation method for a specific category in the classification of remote sensing image,Geoscience and Remote Sensing Symposium. IEEE,978-981.

Zhao Y S. 2013.Principles and methods of remote sensing application analysis. Science Press.

Zhu Z, Woodcock C E. 2012.Object-based cloud and cloud shadow detection in Landsat imagery. Remote Sensing of Environment , 118(6):83-94.

Zhu Z, Woodcock C E. 2014.Automated cloud, cloud shadow, and snow detection in multitemporal Landsat data: An algorithm designed specifically for monitoring land cover change. Remote Sensing of Environment, 152:217-234. 